\title{
RISCO ERGONÔMICO E CONDIÇÃO DE SAÚDE FUNCIONAL EM BANCÁRIOS OPERADORES DE CAIXA
}

\section{Ergonomic risk and functional health condition of bank tellers \\ Riesgo ergonómico y la condición de salud funcional de operadores de caja bancaria}

\author{
Marcos Lenon Matias \\ Universidade do Extremo Sul Catarinense - UNESC - Criciúma (SC) - Brasil \\ Willians Cassiano Longen \\ Universidade do Extremo Sul Catarinense - UNESC - Criciúma (SC) - Brasil
}

\section{RESUMO}

Objetivo: Avaliar o risco ergonômico e a saúde funcional de bancários operadores de caixa. Métodos: Estudo quantitativo e transversal cuja coleta de dados ocorreu nos meses de setembro e outubro de 2015, numa sala reservada na própria agência bancária. Constituiu-se da aplicação do "Censo de Ergonomia", juntamente com questionário com variáveis relacionadas a características da saúde, focado nos sistemas osteomuscular e características ocupacionais, sendo aplicado em caixas bancários de Criciúma e região. Na análise estatística, utilizou-se o programa SPSS, com dados simples expostos em frequência e, para as correlações, utilizou-se o teste Qui-Quadrado, com nível de confiança de 95\%. Resultados: Participaram do estudo 85 trabalhadores ativos, dos quais $57(67,1 \%)$ relataram presença de sintomas de dor/desconforto, sendo que os locais mais acometidos foram: ombro (44; 51,8\%), cervical $(38 ; 44,7 \%)$ e coluna dorso lombar (31; 36,5\%). Não foi encontrada associação entre dor/desconforto e a prática de exercícios nem com o uso de medicamentos para trabalhar. Quanto à presença de inadequações ergonômicas, 43 (50,6\%) relataram presença de inadequações organizacionais; 32 (37,7\%), ambientais; e 23 $(27,1 \%)$, físicas. Encontrou-se correlação entre dor no ombro e inadequação física no ambiente de trabalho ( $\mathrm{p}=0,013)$, bem como com a organizacional $(\mathrm{p}=0,040)$; cervicalgia com inadequação organizacional $(\mathrm{p}=0,012)$, e coluna dorso lombar com inadequações do ambiente de trabalho $(\mathrm{p}=0,044)$. Conclusão: $O$ percentual de caixas bancários com sintomatologia musculoesquelética mostrou-se elevado, caracterizando a atividade como de risco potencializado, especialmente pelas condições ergonômicas organizacionais de trabalho.

Descritores: Transtornos Traumáticos Cumulativos; Trabalhadores; Ergonomia.

\section{ABSTRACT}

Objective: To evaluate the ergonomic risk and functional physical condition of bank tellers. Methods: Quantitative, cross-sectional study, whose data collection happened during the months of September and October 2015, in a reserved room in the very bank branch. It consisted in the application of the "Ergonomics Census", along with a questionnaire addressing variables regarding health characteristics, focusing on the musculoskeletal systems and occupational characteristics, which was applied to bank tellers of Criciuma and region. For the statistical analysis, the software SPSS was adopted, having the simple data displayed in frequency and using, for correlations, the Chi-square test, with a 95\% confidence level. Results: The study evaluated 85 active workers, 57 (67.1\%) of which reported the presence of symptoms of pain/ discomfort, and the most affected locations were: shoulder 44 (44; 51.8\%), cervical (38; 44.7\%) and dorso-lumbar spine (31; 36.5\%). No association was found between pain/discomfort and the practice of exercises, nor between the first and the use of medicines to work. As for the presence of ergonomic inadequacies, 43 (50.6\%) reported the presence of organizational ones; 32 (37.7\%), environmental; and 23, (27.1\%) physical inadequacies. Significant correlations were found between shoulder pain and physical inadequacy in the workplace ( $p=0.013)$, and organizational inadequacy $(p=0.040)$ as well; between neck pain and organizational inadequacy $(p=0.012)$, and between dorso-lumbar spine and inadequacy of the working environment ( $p=0.044)$. Conclusion: The percentage of bank tellers with musculoskeletal symptoms was high, which characterizes the activity as one of potentized risk, especially due to the organizational ergonomic conditions of work.

Descriptors: Cumulative Trauma Disorders; Workers; Ergonomics. 


\section{RESUMEN}

Objetivo: Evaluar el riesgo ergonómico y la salud funcional de operadores de caja bancaria. Métodos: Estudio cuantitativo y transversal cuya recogida de datos se dio en los meses entre septiembre y octubre de 2015 en una sala reservada de la propia agencia bancaria. Se constituyó de la aplicación del "Censo de Ergonomía" y un cuestionario con variables relacionadas con las características de salud, en específico sobre los sistemas osteomuscular y características ocupacionales que fueron aplicados en los operadores de caja bancaria de Criciúma y región. Se utilizó el programa SPSS para el análisis estadístico con datos simples expuestos en frecuencia y se utilizó la prueba Chi-cuadrado para las correlaciones con el nivel de confianza del 95\%. Resultados: Participaron del estudio 85 trabajadores activos de los cuales 57 (67,1\%) relataron síntomas de dolor/incomodidad y los sitios más acometidos fueron el hombro (44; 51,8\%), la cervical (38; $44,7 \%)$ y a la columna dorso lumbar (31; 36,5\%). No se ha encontrado asociación entre dolor/incomodidad y la práctica de ejercicios o el uso de medicación para trabajar. Respecto las inadecuaciones ergonómicas, 43 (50,6\%) relataron inadecuaciones de organización; 32 (37,7\%) del ambiente y $23(27,1 \%)$ de inadecuaciones fisicas. Se encontró relación entre el dolor del hombro y la inadecuación fisica para el ambiente de trabajo $(p=0,013)$ así como la inadecuación de organización $(p=0,040)$; el dolor de la cervical con la inadecuación de organización $(p=0,012)$ y la columna dorso lumbar y las inadecuaciones para el ambiente de trabajo $(p=0,044)$. Conclusión: El porcentaje de operadores de caja bancaria con sintomatología musculo esquelética se ha presentado elevada lo que caracteriza la actividad como riesgo potencial, en especial, por las condiciones ergonómicas de organización de trabajo.

Descriptores: Trastornos de Traumas Acumulados; Trabajadores; Ingeniería Humana.

\section{INTRODUÇÃO}

A Política Nacional de Promoção da Saúde (PNPS) destaca no seu objetivo geral, formalizado no atual documento, a atenção para as condições de trabalho. Este olhar se faz necessário devido ao mundo do trabalho e suas condicionantes representarem aspectos de significativa importância para a saúde humana, a exemplo das outras principais dimensões da vida ${ }^{(1)}$.

Devido à alta competitividade no mercado bancário, o setor vem passando por diversas inovações ${ }^{(2,3)}$. As novas situações a que os trabalhadores bancários são submetidos levam à novas demandas físicas e mentais, favorecendo o aumento de lesões por esforços repetitivos (LER) / doenças osteomusculares relacionadas ao trabalho (DORT) $)^{(4)}$.

Entende-se por LER/DORT distúrbios que afetam os tendões, músculos, ossos, articulações, cartilagens e discos intervertebrais, as quais podem ser de ordem inflamatória ou degenerativa. Essas afecções podem levar, como consequência, ao afastamento da atividade ocupacional, à diminuição da capacidade funcional e a altos custos para os cofres públicos - referentes ao benefício do seguro desemprego e licenças saúde ${ }^{(5)}$.

No Brasil, este índice chega a 48,2\% de todas as patologias relacionadas ao trabalho, e as doenças osteomusculares compreendem uma parte significativamente expressiva desse percentual ${ }^{(6)}$. No ano 2000, no estado da Bahia, o índice de afastamentos chegou a $84,5 \%{ }^{(7)}$.

Estudos realizados demonstram a alta incidência de sintomatologia patológica relacionada a fatores psicológicos e osteomusculares em trabalhadores bancários. A maioria dessas pesquisas foi realizada através da utilização de questionários, os quais foram aplicados em bancários que executavam as mais diversas funções, sendo eles trabalhadores ativos ou que não estavam mais trabalhando ${ }^{(8,9)}$.

No Brasil, há uma norma regulamentadora de Ergonomia criada em 23 de novembro de 1990, pelo Ministério do Trabalho e Emprego, editada pela última vez em 2007, que estabelece os parâmetros definindo as condições ideais de trabalho, conhecida como NR17. Essa norma visa diminuir as patologias relacionadas ao trabalho e proporcionar conforto, segurança e desempenho eficiente aos trabalhadores em suas atividades ${ }^{(10)}$. Percebe-se claramente a deficiência no cumprimento e na fiscalização pelos órgãos responsáveis das recomendações legais da Análise Ergonômica do Trabalho (AET) descritas na NR 17, o que dificulta a conformidade das condições de trabalho(11).

A AET apresenta-se como uma alternativa para a prevenção, levando a um aumento da produtividade e maior satisfação do trabalhador durante suas atividades. Composta por todos os riscos a que o colaborador está exposto durante sua atividade (riscos físicos, mentais e sociais) por meio de uma avaliação dos postos de trabalho ${ }^{(12)}$.

A organização, a demanda excessiva de carga de trabalho, o estresse por cobrança de alcance de metas e a realização de atividades altamente repetitivas são os fatores que afetam a saúde funcional em bancários ${ }^{(12)}$.

A desconfiança da veracidade da presença de alguma patologia pela classe hierárquica superior no setor bancário é evidente segundo uma pesquisa realizada com trabalhadores que sofreram de alguma afecção ${ }^{(12)}$. Os trabalhadores com esses diagnósticos sentem-se deprimidos, angustiados e com sensação de impotência. Essa situação leva a utilização de grandes quantidades de medicamentos sem resultados eficazes, aumentando ainda mais o período de afastamento dos trabalhadores ${ }^{(13)}$.

O tempo prolongado de atividades, aliado aos fatores biopsicossociais aos quais os trabalhadores bancários são expostos, tornam necessário o aprofundamento no conhecimento dos principais motivos desencadeantes de LER/DORT nessa classe. Assim sendo, o objetivo do presente estudo é avaliar o risco ergonômico e a saúde funcional de bancários operadores de caixa. 


\section{MÉTODOS}

Este estudo caracterizou-se como transversal e quantitativo, envolvendo a obtenção de dados resultantes da aplicação de um questionário ergonômico em trabalhadores bancários operadores de caixa.

A amostra foi composta por 85 trabalhadores bancários, segundo dados do Sindicato dos Bancários e Financiários de Criciúma e região. As cidades de Criciúma, Urussanga, Nova Veneza, Içara e Cocal do Sul, em Santa Catarina, possuem aproximadamente 500 trabalhadores bancários, sendo estimado que cerca de 140 exerçam a função de caixa.

Incluíram-se na amostra todos os trabalhadores das agências bancárias que exerciam a atividade de caixa, de ambos os sexos e de diferentes faixas etárias. Foram excluídos da pesquisa os trabalhadores que se encontravam afastados do trabalho ou de férias. Após essa seleção, os questionários eram aplicados em uma sala reservada na própria agência bancária.

O primeiro contato para realização da pesquisa ocorreu através da apresentação do projeto de pesquisa pelo pesquisador e orientador ao presidente e o coordenador da área de saúde do Sindicato dos Trabalhadores Bancários e Financiários de Criciúma e região. Após o aceite deles para o desenvolvimento da pesquisa, entrou-se em contato com os operadores de caixa dentro do perfil, explicou-se a proposta do projeto, e aqueles que aceitaram participar assinaram o Termo de Consentimento Livre e Esclarecido. Em seguida, responderam aos questionários. O estudo percorreu 17 agências bancárias de Criciúma e região entre os meses de setembro e outubro de 2015.

O questionário utilizado no presente estudo foi baseado no instrumento ergonômico de Couto e Cardoso (2007) ${ }^{(14)}$, denominado Censo de Ergonomia. As variáveis avaliadas neste estudo se referiam aos seguintes pontos: nível de escolaridade; tempo de trabalho na empresa; turno de trabalho; tempo das pausas diárias para descanso e alimentação; postura adotada durante o expediente de trabalho; características do ritmo das atividades realizadas; qualidade ambiental do local de trabalho (ruído, temperatura e iluminação); presença de algum desconforto ou dor no corpo; local do desconforto/dor; característica do desconforto/dor; há quanto tempo sente os sintomas; o grau de magnitude do desconforto/dor; se realiza uso de medicamentos ou outro tipo de intervenção para poder trabalhar; e se o colaborador realiza atividades físicas.

Os dados foram tabulados e analisados no programa Statistical Package for the Social Sciencies (SPSS) versão 20.0. A investigação da existência de associação entre as variáveis quantitativas foi realizada por meio da aplicação dos testes quiquadrado de Pearson e razão de verossimilhança, seguidos de análise de resíduo quando observada significância estatística, com nível de confiança de $95 \%$.

Realizou-se o presente estudo após a aprovação do Comitê de Ética em Pesquisa da Universidade do Extremo Sul Catarinense sob parecer $n^{\circ}$ 1.202.806/2015 e a autorização assinada da Carta de Aceite / Termo de Autorização pelo presidente do Sindicato dos Bancários e Financiários de Criciúma e região. Além disso, a pesquisa só foi realizada após a assinatura do Termo de Consentimento Livre Esclarecido pelos trabalhadores das agências bancárias participantes.

\section{RESULTADOS}

A amostra foi composta por 85 operadores de caixas e no perfil sociodemográfico pode-se observar que a maioria era do sexo masculino, com mais de 36 meses nessa atividade e praticantes de atividade física (Tabela I).

Tabela I - Perfil sociodemográfico dos operadores de caixa de Criciúma e região, Santa Catarina, 2015 (n=85).

\begin{tabular}{lc}
\hline Variável & Média \pm DP \\
\hline Idade (anos) & $37,65 \pm 10,94$ \\
Sexo & $\mathrm{n}(\%)$ \\
Masculino & $47(55,3)$ \\
Feminino & $38(44,7)$ \\
Escolaridade & $\mathrm{n}(\%)$ \\
Ensino médio completo & $8(9,4)$ \\
Ensino técnico completo & $2(2,4)$ \\
Ensino superior completo & $75(88,2)$ \\
Tempo de trabalho na Agência & $\mathrm{n}(\%)$ \\
Menos de 12 meses & $3(3,5)$ \\
13 à 36 meses & $8(9,4)$ \\
25 à 36 meses & $5(5,9)$ \\
Mais de 36 meses & $69(81,2)$ \\
Pratica exercício físico & $\mathrm{n}(\%)$ \\
Sim & $57(67,1)$ \\
Não & $28(32,9)$ \\
\hline
\end{tabular}

Legenda: $\mathrm{n}=$ número absoluto de participantes, \%= número relativo, $\mathrm{DP}=$ desvio padrão

A Tabela II retrata o ambiente de trabalho, levando em conta sua estrutura física e a organização de trabalho em que os bancários se inserem durante suas atividades. A maioria dos operadores de caixa considerou que ambientes de trabalho tinham uma boa iluminação, não apresentavam problemas em relação a ruídos, tinham temperatura adequada, sem inadequações físicas e ambientais, e tinham local adequado para almoço e descanso. 
Tabela II - Distribuição dos dados referentes ao ambiente de trabalho, Criciúma e região, Santa Catarina, 2015 (n=85).

\begin{tabular}{|c|c|}
\hline Variável & n (\%) \\
\hline \multicolumn{2}{|l|}{ Iluminação } \\
\hline Boa & $65(76,5)$ \\
\hline Regular & $17(20,0)$ \\
\hline Ruim & $3(3,5)$ \\
\hline \multicolumn{2}{|l|}{ Ruído } \\
\hline Alto & $13(15,3)$ \\
\hline Normal & $64(75,3)$ \\
\hline Baixo & $8(9,3)$ \\
\hline \multicolumn{2}{|l|}{ Temperatura } \\
\hline Gelada & $12(14,1)$ \\
\hline Normal & $64(75,3)$ \\
\hline Quente & $9(10,6)$ \\
\hline \multicolumn{2}{|l|}{ Presença de inadequação - física } \\
\hline Sim & $23(27,1)$ \\
\hline Não & $62(72,9)$ \\
\hline \multicolumn{2}{|c|}{ Presença de inadequação - organização } \\
\hline $\operatorname{Sim}$ & $43(50,6)$ \\
\hline Não & $42(49,4)$ \\
\hline \multicolumn{2}{|c|}{ Presença de inadequação - ambiente } \\
\hline $\operatorname{Sim}$ & $32(37,6)$ \\
\hline Não & $53(62,4)$ \\
\hline \multicolumn{2}{|c|}{ Existe local para almoço e descanso, $n=83$} \\
\hline $\operatorname{Sim}$ & $57(68,7)$ \\
\hline Não & $26(31,3)$ \\
\hline \multicolumn{2}{|l|}{ Postura predominante de trabalho } \\
\hline Sentado & $76(89,4)$ \\
\hline Alternada entre sentado/em pé & $9(10,6)$ \\
\hline Horas trabalhadas, $\mathrm{n}=80$ & $6,45 \pm 0,93$ \\
\hline
\end{tabular}

n: número absoluto de participantes; \%: número relativo; $\mathrm{DP}=$ desvio padrão

A Tabela III apresenta os números em relação à presença de dor/desconforto e os locais onde eles se apresentam. Pode-se observar que $67,1 \%(n=57)$ refere dor/desconforto, sendo o ombro $(51,8 \% ; n=44)$ o local mais acometido.

Na Tabela IV estão apresentados os resultados da correlação entre presença de dor/desconforto versus prática de exercícios, e da correlação do uso de medicamentos para trabalhar versus presença de dor/desconforto.

Finalizando, a Tabela $\mathrm{V}$ apresenta os resultados da correlação da presença de dor/desconforto no ombro versus inadequação física, inadequação organizacional ou inadequação do ambiente. 
Tabela III - Dor e desconforto dos bancários de Criciúma e região, Santa Catarina, 2015 (n=85).

\begin{tabular}{|c|c|}
\hline Variável - presença e locais acometidos & n (\%) \\
\hline Dor/Desconforto & $57(67,1)$ \\
\hline Ombro & $44(51,8)$ \\
\hline Pescoço & $38(44,7)$ \\
\hline Coluna & $31(36,5)$ \\
\hline Punhos & $23(27,1)$ \\
\hline Braços & $19(22,4)$ \\
\hline Mãos & $17(20,0)$ \\
\hline Antebraços & $12(14,1)$ \\
\hline Cotovelos & $8(9,4)$ \\
\hline Quadril & $8(9,4)$ \\
\hline Pernas & $7(8,2)$ \\
\hline Joelhos & $6(7,1)$ \\
\hline Tornozelos/Pés & $6(7,1)$ \\
\hline Coxas & $2(2,4)$ \\
\hline Variável - Característica da dor/desconforto $(n=57)$ & n (\%) \\
\hline Dor & $40(71,9)$ \\
\hline Cansaço & $32(56,1)$ \\
\hline Dolorimento & $23(40,4)$ \\
\hline Formigamento/adormecimento & $20(35,1)$ \\
\hline Perda de força & $16(28,1)$ \\
\hline Limitação de movimento & $12(22,8)$ \\
\hline Estalos & $10(17,5)$ \\
\hline Peso & $9(15,8)$ \\
\hline Choques & $3(5,3)$ \\
\hline
\end{tabular}

Legenda: $\mathrm{n}=$ número absoluto de participantes

Tabela IV - Distribuição quanto à presença de dor ou desconforto nos segmentos corporais versus a utilização de medicamento e versus a pratica de exercício, Santa Catarina, $2015(\mathrm{n}=85)$.

\begin{tabular}{|c|c|c|c|}
\hline & \multicolumn{2}{|c|}{$\begin{array}{c}\text { Sente dor/desconforto em MMSS, } \\
\text { Coluna ou MMII, n (\%) }\end{array}$} & \multirow[t]{2}{*}{ Valor - p } \\
\hline & Sim & Não & \\
\hline \multicolumn{4}{|c|}{ Pratica exercício } \\
\hline Sim & $38(66,7)$ & $19(33,3)$ & 0,913 \\
\hline Não & $19(67,9)$ & $9(32,1)$ & \\
\hline \multicolumn{4}{|c|}{ Utiliza medicamentos } \\
\hline $\operatorname{Sim}$ & $16(94,1)$ & $1(5,9)$ & \\
\hline Não & $24(96,0)$ & $1(4,0)$ & 0,481 \\
\hline Às vezes & $17(100,0)$ & $0(0,0)$ & \\
\hline
\end{tabular}

Legenda: MMSS $=$ membros superiores; $\mathrm{MMII}=$ membros inferiores; $\mathrm{n}=$ número absoluto de participantes; $\%=$ número relativo de participantes. 
Tabela V - Correlação entre Desconforto e Condição Ergonômica de Criciúma e região, Santa Catarina, 2015 (n=85).

\begin{tabular}{|c|c|c|c|}
\hline & \multicolumn{2}{|c|}{ Dor/Desconforto no ombro - n (\%) } & \multirow{2}{*}{ Valor - $\mathbf{p}$} \\
\hline & Sim & Não & \\
\hline \multicolumn{4}{|c|}{ Inadequação física } \\
\hline Sim & $17(73,9)$ & $6(26,1)$ & \multirow{2}{*}{$0,013^{*}$} \\
\hline Não & $27(43,5)$ & $35(56,5)$ & \\
\hline \multicolumn{4}{|c|}{ Inadequação organizacional } \\
\hline Sim & $27(62,8)$ & $16(37,2)$ & \multirow[t]{2}{*}{$0,040^{*}$} \\
\hline Não & $17(40,5)$ & $25(59,5)$ & \\
\hline \multicolumn{4}{|c|}{ Inadequação do ambiente } \\
\hline Sim & $20(62,5)$ & $12(37,5)$ & \multirow[t]{2}{*}{0,124} \\
\hline \multirow[t]{3}{*}{ Não } & $24(45,3)$ & $29(54,7)$ & \\
\hline & \multicolumn{2}{|c|}{ Dor/Desconforto no pescoço-n (\%) } & \multirow{2}{*}{ Valor - $\mathbf{p}$} \\
\hline & Sim & Não & \\
\hline \multicolumn{4}{|c|}{ Inadequação física } \\
\hline Sim & $13(56,5)$ & $10(43,5)$ & \multirow{2}{*}{0,182} \\
\hline Não & $25(40,3)$ & $37(59,7)$ & \\
\hline \multicolumn{4}{|c|}{ Inadequação organizacional } \\
\hline Sim & $25(58,1)$ & $18(41,9)$ & \multirow[t]{2}{*}{$0,012^{*}$} \\
\hline Não & $13(31,0)$ & $29(69,0)$ & \\
\hline \multicolumn{4}{|c|}{ Inadequação do ambiente } \\
\hline Sim & $17(53,1)$ & $15(46,9)$ & \multirow[t]{2}{*}{0,225} \\
\hline \multirow[t]{3}{*}{ Não } & $21(39,6)$ & $32(60,4)$ & \\
\hline & \multicolumn{2}{|c|}{ Dor/Desconforto na coluna - n (\%) } & \multirow{2}{*}{ Valor $-\mathbf{p}$} \\
\hline & Sim & Não & \\
\hline \multicolumn{4}{|c|}{ Inadequação física } \\
\hline Sim & $10(43,5)$ & $13(56,5)$ & \multirow{2}{*}{0,414} \\
\hline Não & $21(33,9)$ & $41(66,1)$ & \\
\hline \multicolumn{4}{|c|}{ Inadequação organizacional } \\
\hline Sim & $20(46,5)$ & $23(53,5)$ & \multirow[t]{2}{*}{0,052} \\
\hline Não & $11(26,2)$ & $31(73,8)$ & \\
\hline \multicolumn{4}{|c|}{ Inadequação do ambiente } \\
\hline Sim & $16(50,0)$ & $16(50,0)$ & \multirow[t]{2}{*}{$0,044^{*}$} \\
\hline Não & $15(28,3)$ & $38(71,7)$ & \\
\hline
\end{tabular}

$\mathrm{n}$ : número absoluto de participantes; \%: número relativo; ${ }^{*} \mathrm{p} \leq 0,05$.

\section{DISCUSSÃO}

Os resultados do presente estudo demonstram uma alta prevalência $(67,1 \%)$ de caixas bancários com dor/desconforto. Os mesmos contam com um tempo relativamente prolongado de atividades na área e em sua maioria são praticantes de atividade física, denotando preocupação dos trabalhadores com sua saúde.

Em relação a esta preocupação com a saúde, nas últimas décadas, vem ganhando força a atitude de cuidar dos condicionantes de vida, de forma a reduzir a vulnerabilidade de adoecer. Tais atitudes contribuem para a redução da vulnerabilidade às doenças crônicas e incapacidade, representando mudança social e, portanto, de saúde geral ${ }^{(1)}$.

Foi demonstrado na atual pesquisa que a maioria dos caixas bancários de Criciúma e região consideram a iluminação do ambiente de trabalho adequada. Para identificar caracteres e detalhes em documentos, é necessário que a iluminação no posto de trabalho esteja na intensidade ideal ${ }^{(14)}$ e os níveis ideais de iluminação variam de acordo com o tipo de trabalho realizado e idade do trabalhador. A iluminação ideal para ambientes informatizados deve ser entre 450 e 550 lux, e atividades em condições inadequadas de iluminação podem levar a fadiga visual, caracterizada por ardor, dolorimento dos olhos, vermelhidão da conjuntiva, modificação na frequência de piscar, lacrimejamento, fotofobia, diplopia, sensação de visão velada, entre outras alterações ${ }^{(14,15)}$.

Outro aspecto analisado no presente estudo foi a temperatura e o ruído do ambiente, e a maioria dos participantes considerou a temperatura $(75,3 \%)$ e o ruído $(75,3 \%)$ do local de trabalho adequados. A temperatura ideal para o trabalho é de 20 a $23^{\circ} \mathrm{C}$, e quanto mais quente a temperatura no ambiente de trabalho, menor será a tolerância para atividades físicas e mentais do 
trabalhador ${ }^{(16)}$. O ser humano tem a capacidade de se adequar às diferentes temperaturas, mas nem todas as condições são consideradas adequadas, o que leva à diminuição da efetividade nas atividades realizadas ${ }^{(16)}$.

Os estímulos auditivos, que não contribuem positivamente para o desempenho ideal de uma atividade, definem o termo ruído. O nível ideal para trabalhos que exigem maior concentração, como o trabalho em postos de trabalho informatizados, é de 65 decibéis ${ }^{(17)}$.

Os resultados da presente pesquisa mostram convergência com o encontrado em uma publicação envolvendo as cargas de trabalho do tipo organizacionais e psicossociais como sendo as que resultam como maior influência no adoecimento da população estudada. Entre as variáveis inclusas na carga de trabalho do tipo organizacional, as que geram maior prejuízo à saúde dos trabalhadores são: atividades estressantes, remuneração inadequada, insegurança no emprego, desvalorização do trabalho, posturas da chefia, exames médicos do banco insatisfatórios e disputas entre os colegas ${ }^{(12)}$.

A média de horas trabalhadas pelos caixas bancários do estudo em questão é de 6,45 horas, sendo que a maior parte desses trabalhadores exerce suas atividades na posição sentada. Um trabalho realizado num município do interior do estado do Rio Grande do Sul, a média de horas trabalhadas dos entrevistados foi de 7,28 horas ${ }^{(18)}$. O desgaste físico e mental derivados de atividades com grandes somas de dinheiro, em que há necessidade de concentração elevada e a permanência da postura sentada em maior parte da jornada de trabalho, representa situação que pode levar a lesões irreversíveis da coluna vertebral, justificando a redução da jornada de trabalho dos bancários de oito para seis horas ${ }^{(19)}$.

Grande parte da amostra da atual pesquisa relatou possuir algum sintoma de dor/desconforto, cujos resultados encontram-se superiores quando comparados com as pesquisas prévias realizadas com bancários em geral nas cidades de Criciúma (34,18\% de 158 bancários de agências públicas e privada) e de Porto Alegre (27,5\% de 356 bancários) nos anos de 2003 e 2012 , respectivamente ${ }^{(7,8)}$.

Pôde-se observar na atual investigação que os principais segmentos corporais acometidos foram: ombro, cervical, coluna e punho; e os principais tipos de sintomas são: dor, cansaço, dolorimento e formigamento/adormecimento. Um recente estudo realizado com bancários em município de médio porte no interior do Paraná, encontrou os mesmos segmentos corporais com sintomatologias similares ${ }^{(20)}$.

Há registro de que, após a realização de exercícios físicos durante a jornada de trabalho, há diminuição de queixas osteomusculares pelos trabalhadores ${ }^{(21)}$. Como foi observado no presente estudo, apesar do resultado da presença de sintomas de LER/DORT, a prática de exercício físico apresentou-se elevada, mas não houve associação estatisticamente significativa entre esses fatores.

Ao se correlacionar os resultados das variáveis, presença de dor/desconforto versus uso de medicamentos para trabalhar, não se encontrou significância estatística no atual estudo, o que está em desacordo para com estudo realizado com 1.117 bancários, em que 49,9\% afirmaram utilizar algum tipo de medicamento. A discrepância entre os estudos pode ser explicada pelo fato de que as populações de bancários foram diferentes, em estados diferentes. Além disso, diferentemente do presente estudo, que avaliou somente a função caixa, num estudo comparativo foram avaliados os trabalhadores de todas as funções do banco $^{(22)}$.

No presente estudo, quando correlacionados os resultados da variável presença de dor/desconforto no ombro versus a presença de inadequação física, organizacional e ambiental, observou-se que há associação significativa entre essas variáveis. Em estudo com características similares a esta pesquisa foi possível observar que os postos de trabalho avaliados possuíam características ergonômicas físicas excelentes, porém 11\% da amostra foi diagnosticada com DORT ${ }^{(23)}$.

Os resultados da correlação entre as variáveis presença de dor/desconforto no pescoço versus presença de inadequação física, organizacional e ambiental do atual estudo demonstrou que há associação significativa entre os sintomas de dor/ desconforto na região do pescoço e a presença de inadequação organizacional do trabalho. Um estudo realizado em 2007 com caixas bancários, revelou forte correlação entre situações problemáticas no ambiente de trabalho envolvendo características físicas e organizacionais versus dor/desconforto/peso/dormência ou limitação dos movimentos e dor/ queimação nas costas/ $\operatorname{pescoço}^{(23)}$.

Foi encontrada, da mesma forma, associação significativa quando correlacionada a presença de dor/desconforto na coluna versus presença de inadequação do ambiente de trabalho na presente pesquisa. Em uma pesquisa realizada em postos de trabalho informatizados, os autores sugeriram que há discreta associação entre inadequações ambientais versus diminuição da funcionalidade da coluna cervical ${ }^{(24)}$. O que mostra problema futuro para a saúde do trabalhador.

Como limitação do presente estudo, tem-se as respostas autorreferidas, que são dependentes da percepção dos participantes a respeito dos fenômenos avaliados.

O trabalho de caixa bancário apresenta uma criticidade especial, sendo atividade que merece atenção quanto à criação e fortalecimento das políticas de cuidado da saúde e segurança ocupacional, incluindo especialmente empresas bancárias, públicas ou privadas em suas formas de organização, valorizar a qualidade de vida no trabalho e afastar-se dos limiares perigosos de esgotamento psicofisiológico. 


\section{CONCLUSÃO}

Os trabalhadores bancários que exercem a função de caixa nas cidades avaliadas apresentam índices elevados de sintomas de dor ou desconforto, sendo que os segmentos corporais mais atingidos por afecções são os membros superiores e a coluna.

Neste estudo pôde-se observar, da mesma forma, que as características físicas, ambientais e, especialmente, as organizacionais do trabalho estão relacionadas à presença dos sintomas, segundo a percepção dos trabalhadores. Além disso, a presença de inadequações referente à organização do trabalho teve destaque.

\section{REFERÊNCIAS}

1. Ministério da Saúde (BR). Política Nacional de Promoção da Saúde. $3^{\mathrm{a}}$ ed. Brasília: Ministério da Saúde; 2010. (Série B. Textos Básicos de Saúde. Série Pactos pela Saúde, v. 7).

2. Moraes PWT, Bastos AVB. Os sintomas de LER/DORT: um estudo comparativo entre bancários com e sem diagnóstico. Psicol Ciênc Prof. 2017;37(3):624-37.

3. Silva LS, Navarro VL. organização do trabalho e saúde de trabalhadores bancários. Rev Latinoam Enferm. 2012;20(2): 226-34.

4. Brasil. Instrução Normativa INSS/DC n ${ }^{0} 98$ de 05 de dezembro de 2003. Aprova Norma Técnica sobre Lesões por Esforços Repetitivos- LER ou Distúrbios Osteomusculares Relacionados ao Trabalho- DORT. Diário Oficial União; Brasília. 10 dez 2003.

5. Souza NSS, Santana VS. incidência cumulativa anual de doenças musculoesqueléticas incapacitantes relacionadas ao trabalho em uma área urbana do Brasil. Cad. Saúde Pública [online]. 2011;27(11):2124-34.

6. Ministério da Previdência Social (BR). Informações estatísticas gerais da Previdência Social [acesso em 2016 Mar 31 ]. Disponível em: http://creme.dataprev. gov.br/infologo/GCON/Ver07.php

7. Souza NSS, Santana VS, Albuquerque-Oliveira PR, Barbosa-Branco A. Doenças do trabalho e benefícios previdenciários relacionados à saúde, Bahia, 2000. Rev Saúde Pública. 2008; 42(4):630-8.

8. Nunes MS, Longen, WC. Incidência de alterações relacionadas à LER/DORT: uma análise do fenômeno junto aos bancários de Criciúma- Santa Catarina. Criciúma: Universidade do Extremo Sul Catarinense; 2003.

9. Scopel J, Oliveira PAB, Wehrmeister FC. LER/DORT na terceira década da reestruturação bancária: novos fatores associados? Rev Saúde Pública. 2012;46(5):875-85.

10. Ministério do Trabalho e Emprego (BR). Norma Regulamentadora 17 - Ergonomia. Brasília: TEM; 2007.

11. Jackson JM Filho, Lima FPA. Análise Ergonômica do Trabalho no Brasil: transferência tecnológica bem-sucedida? Rev Bras Saúde Ocup. 2015;40(131):12-7.

12. Marques GS, Giongo CR. Trabalhadores Bancários em Sofrimento: uma análise da literatura nacional. Rev Psicol Organ Trab. 2016;16(3):220-35

13. Ragadali A Filho, Leal I, Anjos QS, Leite SA, Danilussi DP. Lesões por Esforços Repetitivos (LER): uma doença misteriosa do trabalho. Rev Saberes. 2015;3(2):76-89.

14. Couto HA, Cardoso OS. Censo de ergonomia. Belo Horizonte: Ergo; 2007.

15. Garlet E, Santos LA, Perufo LD, Godoy LP, Marzall LF. A iluminação natural como fator de desempenho em ambientes industriais. Rev Adm UFSM. 2015;8(Esp):24-34.

16. Pontes ALR. Influência do projeto das aberturas no conforto térmico de apartamentos e residências unifamiliares [trabalho de conclusão de curso]. Curitiba: Universidade Federal Tecnológica do Paraná; 2014.

17. Farias VHV, Buriti AKL, Rosa MRD. ocorrência de perda auditiva induzida pelo ruído em Carpinteiros. Rev CEFAC. 2012;14(3):413-22.

18. Sznelwar LI. Saúde dos bancários. São Paulo: Publicher; 2011.

19. Rodrigues AF, Quilião PL, Pinheiro LSM, Carneiro CMG, Carneiro CFG, Peres DPS. Identificação do uso de álcool em bancários. SMAD Rev Eletrônica Saúde Mental Álcool Drog. 2016;12(4):207-13.

20. Barreto MAA. Análise sobre a jornada de trabalho dos bancários: a discussão sobre o exercício de cargos de confiança, horas extraordinárias. Âmbito Jurídico. Jan. 2012. 
21. Oliveira RA, Souza STM. Lesões por esforços repetitivos / distúrbios osteomusculares relacionados à atividade bancária. Rev Elet Sistemas Gestão. 2015;10(1):124-32.

22. Martins LV, Baú LMS, Marziale MHP, Franco BAS. Exercícios físicos e seus efeitos nas queixas osteomusculares e na satisfação do trabalho. Rev Enferm UERJ. 2011;19(4):587-91.

23. Gaviraghi D, Antoni CD, Amazarray MR, Schaefer LS. Medicalização, uso de substâncias e contexto de trabalho em bancários do Rio Grande do Sul, Brasil. Rev Psicol Organ Trab. 2016;16(1):61-72.

24. Luís BCC, Scarsi CN, Longen WC. Regulações posturais relacionadas ao uso de microcomputadores no trabalho administrativo: Relações com a disfunção da coluna cervical. Rev Inova Saúde. 2014;3(1):108-25.

\section{Endereço para correspondência:}

Marcos Lenon Matias

Universidade do Extremo Sul Catarinense - UNESC

Av. Universitária, 1105

Bairro Universitário

CEP 88806-000 - Criciúma - SC- Brasil

E-mail: markinhoslmatias@hotmail.com 